\title{
Sawflies of Southern part of Somogy county (Hymenoptera: Symphyta)
}

\author{
AtTIla Haris \\ H-1076 Budapest Garay u. 19. 2/20., Hungary, email: attilaharis@yahoo.com,
}

HARIS, A.: Sawflies of Southern part of Somogy county (Hymenoptera: Symphyta).

Abstract: 100 species of 683 specimens were collected in the Duna-Dráva National Park and the adjacent territory: Csokonyavisonta Wooded Pasture. Caliroa cothurnata (Serville, 1823) is new record for Hungary and for the Carpathian Basin. Rare species are: Xyela (Xyela) julii (Brébisson, 1818), Monoctenus juniperi (Linné, 1758), Dolerus (Poodolerus) blanki Liston, 1995 and Euura fuscomaculata (Förster, 1854). Aproceros leucopoda Takeuchi, 1939 is a newly introduced invasive species.

Keywords: Hymenoptera, Symphyta, Somogy, Hungary, new record

\section{Introduction}

This year, the Southern part of Somogy County (Fig. 1) was investigated, namely the Somogy part of Danube-Dráva National Park and the protected wooded pasture at Csokonyavisonta. The sampling places are restricted to 3 different areas. These are the Protected Wooded pasture at Csokonyavisonta, Juniper woodlands at Darány and the floodplain of River Dráva at Vízvár.

\section{Csokonyavisonta Protected Wooded Pasture}

This wooded pasture (Figs. 2, 5 and 6) was a result of human activity, namely the traditional extensive livestock breeding (cattle and pigs) in the last centuries.

The altitude of the area varies between 120-140 m. Within this small area, there are high diversity of habitats typical for Somogy county from the sand dunes till the wet and swampy depressions holding alder-moors.

Among the forest associations there are hornbeam-oak, Turkey oak forests, alder bogs and also gallery forests.

By giving up grazing, the proportion of open areas has been steadily declining. Maintaining the remaining grasslands is an important task for nature conservation.

\section{Juniper woodland at Darány}

Juniper woodland (Figs. 3 and 4) is also a result of pasture farming: grazing animals avoided prickly junipers therefore very special and unique habitat is formed in this area during the last centuries. For our days, only two larger spots remained, the other parts were replaced by forest, when grazing was abandoned. These spots are actually sand dunes covered by sporadic Juniper groups. 


\section{Floodplain forests of River Dráva}

River Dráva originates in the Tyrolean Alps and reaches the territory of Hungary at Örtilos. The fast fall of the flow of the river brings with itself lot of sediments. Its fall is gradually decreasing on the Hungarian part, and its sediments are being deposited. The characteristic formations of the Dráva are the reef islands, which are constantly being built and destroyed, thus always changing their locations. The shore is accompanied by floodplain groves, where white willow, white poplar and black poplar are the canopylevel trees. Beyond the floodplain, the next stage of succession is the oak-ash-elm forest in the higher areas.

From this region ZomBori (1985) published a paper titled Data to the knowledge of Symphyta fauna of the Barcs juniper woodland. Further faunistic data were published in HARIS 2001b and ROLLER \& HARIS 2008.

\section{Material and methods}

During the 2020 collecting season, I spent 14 days with collecting in April, 9 days in May, 2 days in July and 1 day in August.

The regular rainfall and the overcast weather conditions made the collecting very difficult in May. The applied method was net sweeping from the beginning till the first decade of May. After this, the capture of individuals was the dominant method combined with netsweeping of lower canopies and bushes.

For identification, Zhelochovtsev's work on the sawflies of the European part of the former USSR and the latest Slovakian monograph (ZHELOCHOVTSEV 1988, MACEK et al., 2020) were consulted. We also used some recent revisions and works to make the identifications even more precise (ACHTERBERG \& AARTSEN 1986, HARIS 2006, KoCH 1988, Prous et. al. 2014, ZOMBORI 2016).

For the discussion of the distribution of sawflies, we consulted the book of Roller and Haris titled Sawflies of the Carpathian Basin, History and Current Research (RoLLER \& HARIS 2008), the most recent European checklist of species (TAEGER et al. 2006) augmented by other faunistic records from the Carpathian Basin (MocsáRY 1900, Roller 1993, 1994, 1996, 1998, 1999a, b, c, d, e, 2000a, b, c, 2001, 2004, 2005, 2006a, b; 2010, Roller \& LuKáš 1999, Roller et. al. 2006, Roller \& MACEK 2017, Roller \& OLŠOVSKÝ 2012, HaRIS 2001a, 2009, 2010, 2011, 2012, 2018a,b; HARIS \& GYURKOVICS 2012).

The higher classification of sawflies applied in this work follows the Hymenoptera part of Fauna Europaea (ACHTERBERG 2013).

\section{List of sites}

Babócsa: Basakert, between $46^{\circ} 2^{\prime} 28.80^{\prime \prime} \mathrm{N}, 1^{\circ} 21^{\prime} 27.54^{\prime \prime} \mathrm{E}$ and $46^{\circ} 2^{\prime} 35.89^{\prime \prime} \mathrm{N}, 1^{\circ} 21^{\prime} 35.33^{\prime \prime} \mathrm{E}$, altitude 118 $119 \mathrm{~m}$.

Babócsa: Két-árok köze, between $46^{\circ} 2^{\prime} 22.17^{\prime \prime} \mathrm{N}, 17^{\circ} 21^{\prime} 11.25^{\prime \prime E}$ and $46^{\circ} 2^{\prime} 33.01^{\prime \prime N}, 1^{\circ} 21^{\prime} 17.35^{\prime \prime E}$, altitude: 109-111 m.

Bolhó: Libalegelö, between $46^{\circ} 2^{\prime} 17.66^{\prime \prime} \mathrm{N}, 17^{\circ} 18^{\prime} 17.80^{\prime \prime} \mathrm{E}$ and $46^{\circ} 2^{\prime} 13.13^{\prime \prime} \mathrm{N}, 1^{\circ} 18^{\prime} 25.88^{\prime \prime} \mathrm{E}$, altitude: $106-$ $107 \mathrm{~m}$.

Darány: Kecskésrét: Juniper woodland, between $45^{\circ} 58^{\prime} 47.15^{\prime \prime} \mathrm{N}, 17^{\circ} 34^{\prime} 37.32^{\prime \prime} \mathrm{E}$ and $45^{\circ} 58^{\prime} 53.56^{\prime \prime} \mathrm{N}$, 17³4'44.93"E, altitude: $127-129 \mathrm{~m}$.

Darány: Kis-Rigócz: Juniper woodland (Fig. 4), between $45^{\circ} 58^{\prime} 43.11^{\prime \prime N}, 17^{\circ} 34^{\prime} 15.17^{\prime \prime} \mathrm{E}$ and $45^{\circ} 58^{\prime} 42.61^{\prime \prime N}$, 17³3'49.49"E, altitude: $127-130 \mathrm{~m}$.

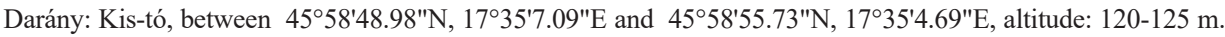




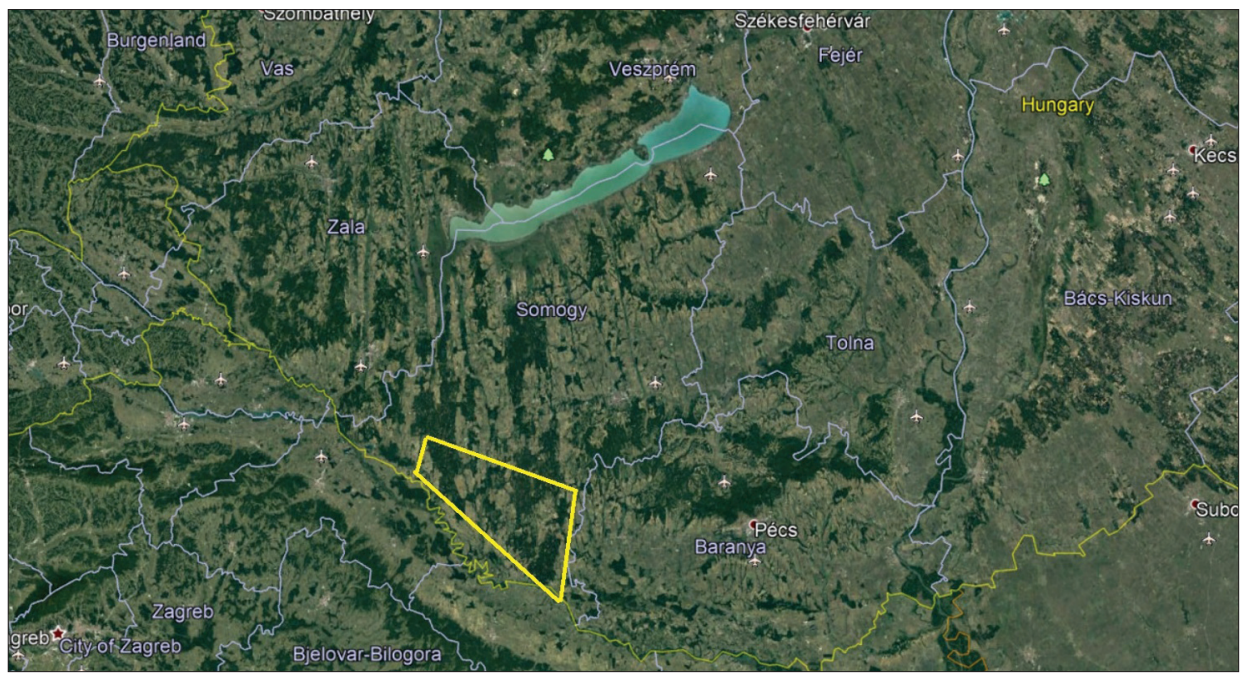

Fig. 1: The investigated area

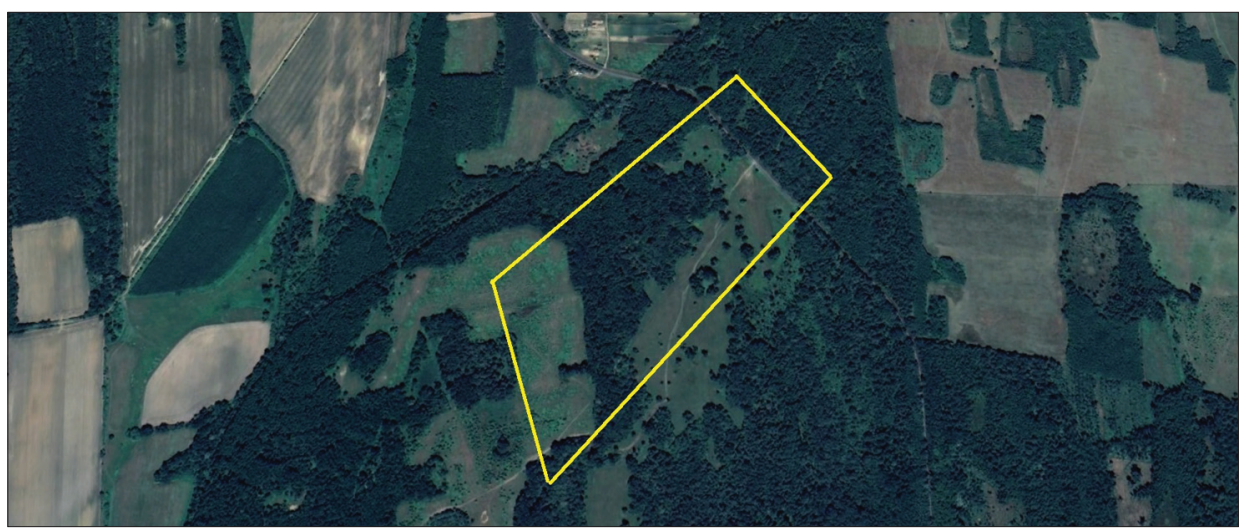

Fig. 2: Map of the protected wooded pasture at Csokonyavisonta

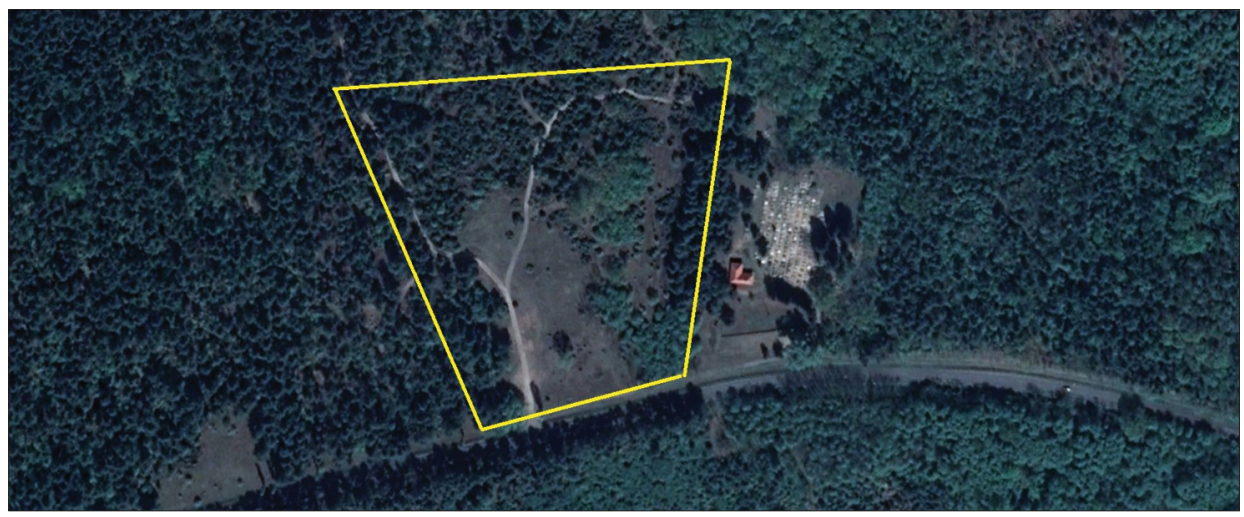

Fig. 3: Map of Darány: Kecskésrét, juniper woodland 
Drávatamási: Old Railway, between $45^{\circ} 57^{\prime} 44.45^{\prime \prime N}, 17^{\circ} 35^{\prime} 10.05^{\prime \prime E}$ and 4557'39.31"N, 17³5'18.90"E, altitude: $120-122 \mathrm{~m}$.

Csokonyavisonta: Protected Wooded Pasture (Figs. 5 and 6), between $46^{\circ} 3^{\prime} 18.40^{\prime \prime N}, 17^{\circ} 27^{\prime} 29.01^{\prime \prime} \mathrm{E}$ and $46^{\circ}$ 2'55.95"N, 17²6'59.97"E, altitude: $129-138 \mathrm{~m}$.

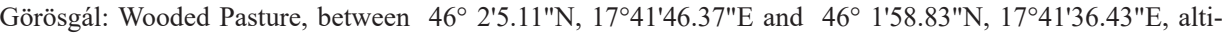
tude: $116-118 \mathrm{~m}$.

Péterhida: Protected Wooded Pasture, between $46^{\circ} 0^{\prime} 42.58^{\prime \prime} \mathrm{N}, 17^{\circ} 22^{\prime} 26.52$ "E and $46^{\circ} 00^{\prime} 25.03^{\prime \prime} \mathrm{N}$, $17^{\circ} 22^{\prime} 18.42 " \mathrm{E}$, altitude: $105-107 \mathrm{~m}$.

Vízvár: Dráva-part (Fig. 7), Táborhely, between $46^{\circ} 5^{\prime} 14.60 " \mathrm{~N}, 17^{\circ} 13^{\prime 2} 28.66^{\prime \prime} \mathrm{E}$ and $46^{\circ} 4^{\prime} 59.19^{\prime \prime} \mathrm{N}$, $17^{\circ} 13^{\prime} 40.73$ "E, altitude: $109-115 \mathrm{~m}$.

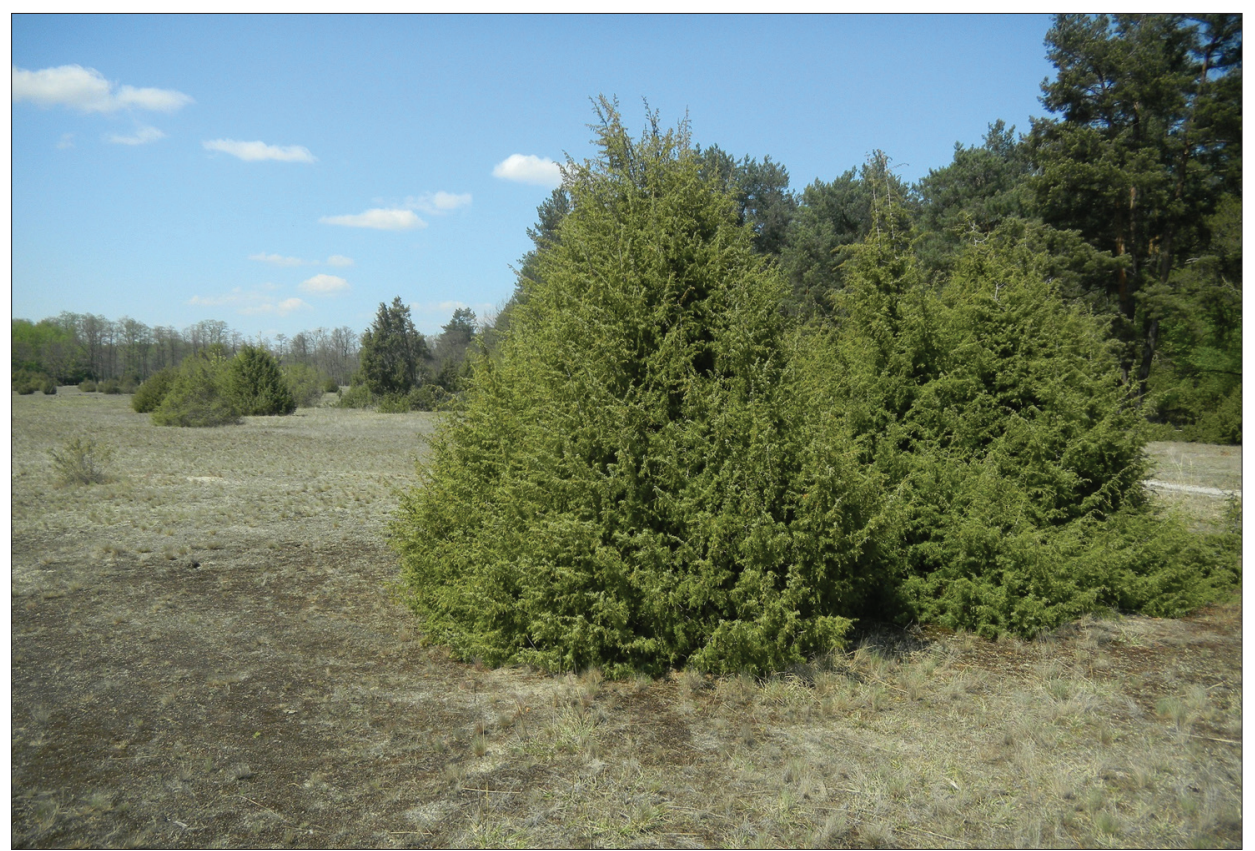

Fig. 4: Juniper woodland at Darány

\section{List of species}

\section{Xyelidae}

Xyela (Xyela) julii (Brébisson, 1818): Darány: Kecskésrét: Juniper woodland, 10. 04. 2020, 1 female. Rare in Hungary. Larva on Pinus sylvestris, P. nigra and P. cembra.

\section{Pamphiliidae}

Pamphilius vafer (Linné, 1767): Babócsa: Két-árok köze, 09. 05. 2020, 1 female, 16. 05. 2020, 3 females. On Alnus glutinosa and A. incana. Sporadic.

\section{Megalodontesidae}

Megalodontes plagiocephalus (Fabricius, 1804): Csokonyavisonta: Protected Wooded Pasture, 16. 05. 2020, 2 females. One of the most frequent Megalodontesidae species. Known hostplant: Peucedanum alsaticum. 


\section{Diprionidae}

Monoctenus juniperi (Linné, 1758): Darány: Kecskésrét: Juniper woodland, 16. 04. 2020, 1 male, 10. 04. 2020, 1 female; Darány: Kis-Rigócz: Juniper woodland, 17. 04. 2020, 3 males, 1 female. Host plants: Juniperus communis and J. nana. Sporadic.

\section{Argidae}

Arge cyanocrocea (Forster, 1771): Csokonyavisonta: Protected Wooded Pasture, 02. 05. 2020, 2 females, 04. 05. 2020, 1 female, 24. 04. 2020, 1 male; Babócsa: Két-árok köze, 02. 05. 2020, 1 female, 23. 05. 2020, 1 male, 19. 07. 2020, 4 females; Babócsa: Basakert, 02. 08. 2020, 1 female; Vízvár: Riverside, 24. 04. 2020, 1 female, 1 male, 23. 05. 2020, 1 male; Bolhó: Libalegelö, 19. 07. 2020, 4 females, 02. 08. 2020, 1 female; Darány: Kis-tó, 19. 07. 2020. Common species. Known host plants: Rubus idaeus and Sanguisorba officinalis.

Arge enodis (Linné, 1767): Bolhó: Libalegelö, 02. 08. 2020, 4 females, 3 males. Frequent, locally common. Larvae on smooth leaved willows like Salix fragilis, Salix alba and Salix purpurea.

Arge melanochra (Gmelin, 1790): Babócsa: Basakert, 02. 08. 2020, 1 male; Bolhó: Libalegelö, 02. 08. 2020, 1 male; Péterhida: Protected Wooded Pasture, 02. 08. 2020, 1 female. Generally the commonest Argid sawfly, however, not common in this region. Hostplant: Crataegus oxycantha.

Arge pagana ssp. pagana (Panzer, 1797): Csokonyavisonta: Protected Wooded Pasture, 02. 05. 2020, 1 female, 22. 04. 2020, 1 male. Frequent. Host plants: Rosa spp. Arge ustulata (Linné, 1758): Csokonyavisonta: Protected Wooded Pasture, 22. 04. 2020, 1 male, 24. 04. 2020, 1 male. Frequent. Larva on Betula, Salix and Crataegus. Aproceros leucopoda Takeuchi, 1939: Vízvár: Riverside, 24. 04. 2020, 1 female. Larva on Ulmus spp. like Ulmus glabra, U. pumila, U. japonica, U. minor and U. laevis. Introduced species. Sporadic, locally frequent.

Sterictiphora angelicae (Panzer, 1799): Bolhó: Libalegelö, 26. 07. 2020, 1 male. Frequent. Larva on Prunus spinosa and Rubus spp.

\section{Cephidae}

Calameuta (Calameuta) filiformis (Eversmann, 1847): Vízvár: Riverside, 24. 04. 2020, 1 female, 09. 05. 2020, 2 males; Csokonyavisonta: Protected Wooded Pasture, 11. 05. 2020, 1 female; Babócsa: Két-árok köze, 16. 05. 2020, 1 female. Generally common species. Larva lives in stems of Arrhenaterum elatius, Phalaris arundinacea, Calamagrostis epigeios, Elytrigia repens and Phragmites communis.

Calameuta (Calameuta) haemorrhoidalis (Fabricius, 1781): Csokonyavisonta: Protected Wooded Pasture, 18. 04. 2020, 1 female, 03. 05. 2020, 1 female; Vízvár: Riverside, 24. 04. 2020, 1 female. Frequent. Hostplant unknown.

Calameuta (Calameuta) pallipes (Klug, 1803): Csokonyavisonta: Protected Wooded Pasture, 09. 05. 2020, 1 female, 1 male; Vízvár: Riverside, 24. 04. 2020, 1 male. Frequent on diverse Poaceae.

Calameuta (Calameuta) punctata (Klug, 1803): Csokonyavisonta: Protected Wooded Pasture, 26. 04. 2020, 1 female; Babócsa: Két-árok köze, 09. 05. 2020, 1 female. Rare. Host plant unknown.

Cephus nigrinus Thomson, 1871: Csokonyavisonta: Protected Wooded Pasture, 02. 05. 2020, 1 female, 23. 04. 2020, 1 male, 04. 05. 2020, 1 male. Frequent species. Host plants: Milium effusum and Poa pratensis. 


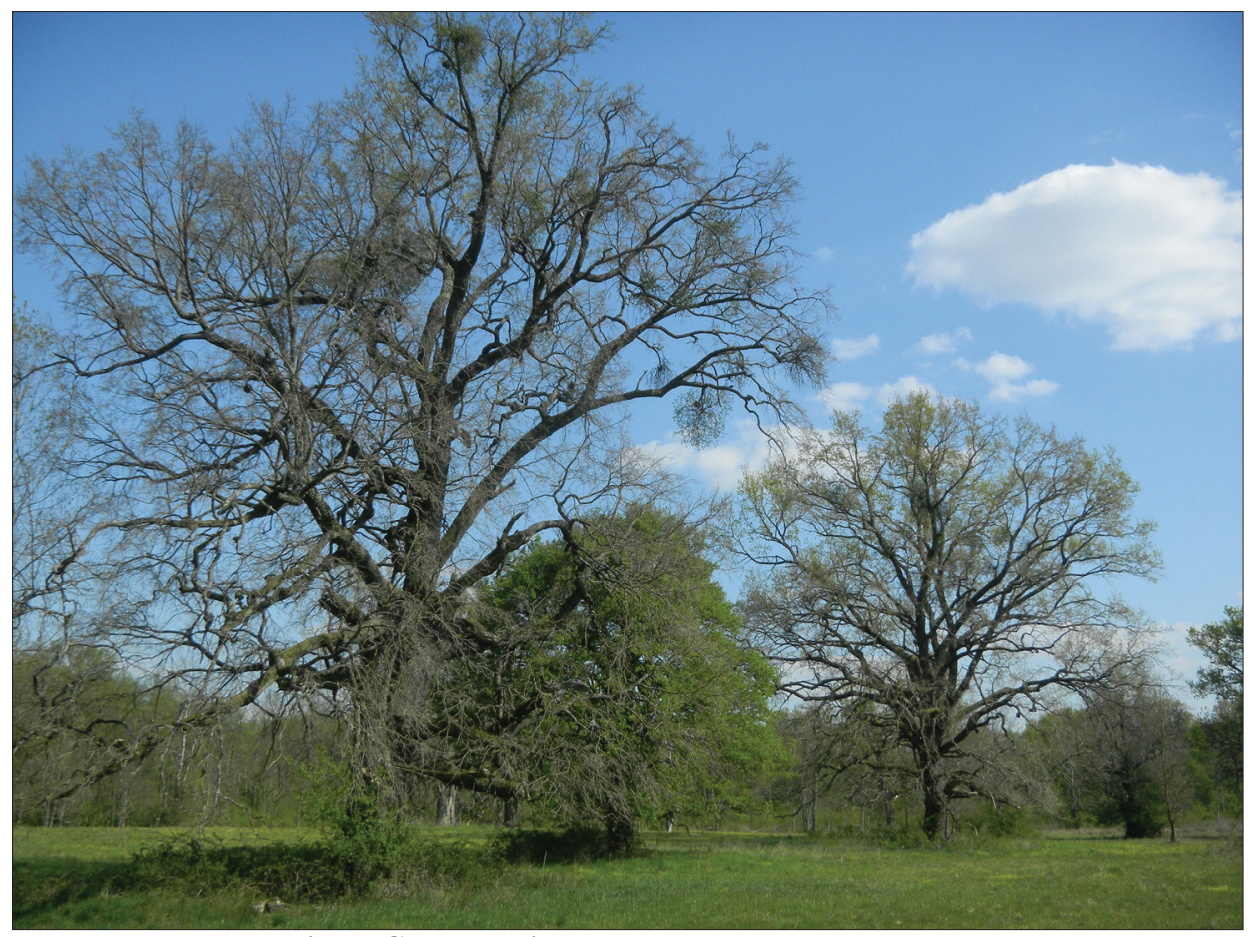

Fig. 5: Csokonyavisonta protected wooded pasture

Cephus pygmus (Linné, 1767): Csokonyavisonta: Protected Wooded Pasture, 11. 05. 2020, 1 female; Babócsa: Két-árok köze, 16. 05. 2020, 1 female, 23. 05. 2020, 1 male. Common. Insect pest of cereals and Gramineae.

Cephus spinipes (Panzer, 1800): Csokonyavisonta: Protected Wooded Pasture, 30. 05. 2020, 5 females, 11. 05. 2020, 1 female, 16. 05. 2020, 1 female, 1 male, 23. 05. 2020, 1 female, 3 males, 09. 05. 2020, 1 male, 30. 05. 2020, 1 male. Frequent. Hostplant: Phleum pratense.

Janus compressus (Fabricius, 1793): Csokonyavisonta: Protected Wooded Pasture, 02. 05. 2020, 1 male. Frequent insect pest of Pyrus and Malus spp.

\section{Tenthredinidae}

Dolerinae

Dolerus (Dolerus) aericeps Thomson, 1871: Péterhida: Protected Wooded Pasture, 02. 08. 2020, 1 female. Frequent. Larva on Equisetum palutre and E. arvense.

Dolerus (Poodolerus) anthracinus (Klug, 1818): Csokonyavisonta: Protected Wooded Pasture, 11. 04. 2020, 1 female. Sporadic. Host plants: Graminae.

Dolerus (Poodolerus) blanki Liston, 1995: Csokonyavisonta: Protected Wooded Pasture, 17. 04. 2020, 1 female. Rare. Hostplant unknown.

Dolerus (Poodolerus) brevicornis Zaddach, 1859: Görösgál: Wooded Pasture, 09. 04. 2020, 1 male. Sporadic. Hosts: Carex cespitosa, C. nigra and some other Carex spp.

Dolerus (Oncodolerus) eversmanni W.F. Kirby, 1882: Darány: Kis-tó, 18. 04. 2020, 1 male. Frequent. Larva on Equisetum arvense and E. palustre. 
Dolerus (Dolerus) germanicus ssp. germanicus (Fabricius, 1775): Babócsa: Két-árok köze, 02. 05. 2020, 1 female, 1 male; Babócsa: Basakert, 02. 08. 2020, 1 female. Common. Larva on Equisetum arvense and E. palustre.

Dolerus (Poodolerus) niger (Linné, 1767): Csokonyavisonta: Protected Wooded Pasture, 23. 04. 2020, 1 female. Sporadic. Host plants: Poaceae.

Dolerus (Poodolerus) nigratus (O.F. Müller, 1776): Csokonyavisonta: Protected Wooded Pasture, 09. 04. 2020, 1 female, 2 males, 10. 04. 2020, 3 females, 1 male, 11. 04. 2020, 1 female, 12. 04. 2020, 2 females, 1 male, 19. 04. 2020, 2 females, 23. 04. 2020, 1 female, 17. 04. 2020, 3 females, 02. 05. 2020, 2 females, 03. 05. 2020, 1 female, 09. 05. 2020, 1 female, 22. 04. 2020, 2 males, 24. 04. 2020, 2 males, 16. 04. 2020, 2 males; Vízvár: Riverside, 24. 04. 2020, 1 male; Darány: Kis-tó, 26. 04. 2020, 1 male. Common. Larva on Gramineae including cereals.

Dolerus (Poodolerus) nitens Zaddach, 1859: Csokonyavisonta: Protected Wooded Pasture, 11. 04. 2020, 1 female, 17. 04. 2020, 1 female. Sporadic. Larva on Cyperaceae and Graminae.

Dolerus (Poodolerus) picipes (Klug, 1818): Csokonyavisonta: Protected Wooded Pasture, 11. 05. 2020, 2 females, 10. 05. 2020, 1 female, 04. 05. 2020, 1 female, 09. 05. 2020, 3 females. Frequent. Larva on Graminae.

Dolerus (Poodolerus) puncticollis Thomson, 1871: Görösgál: Wooded Pasture, 09. 04. 2020, 1 female; Csokonyavisonta: Protected Wooded Pasture, 09. 04. 2020, 1 female, 12. 04. 2020, 1 female, 03. 05. 2020, 1 female. Common. Larva on Graminae including cereals.

Dolerus (Poodolerus) sanguinicollis (Klug, 1818): Vizvár: Riverside, 24. 04. 2020, 1 male. Sporadic. Larva on Gramineae.

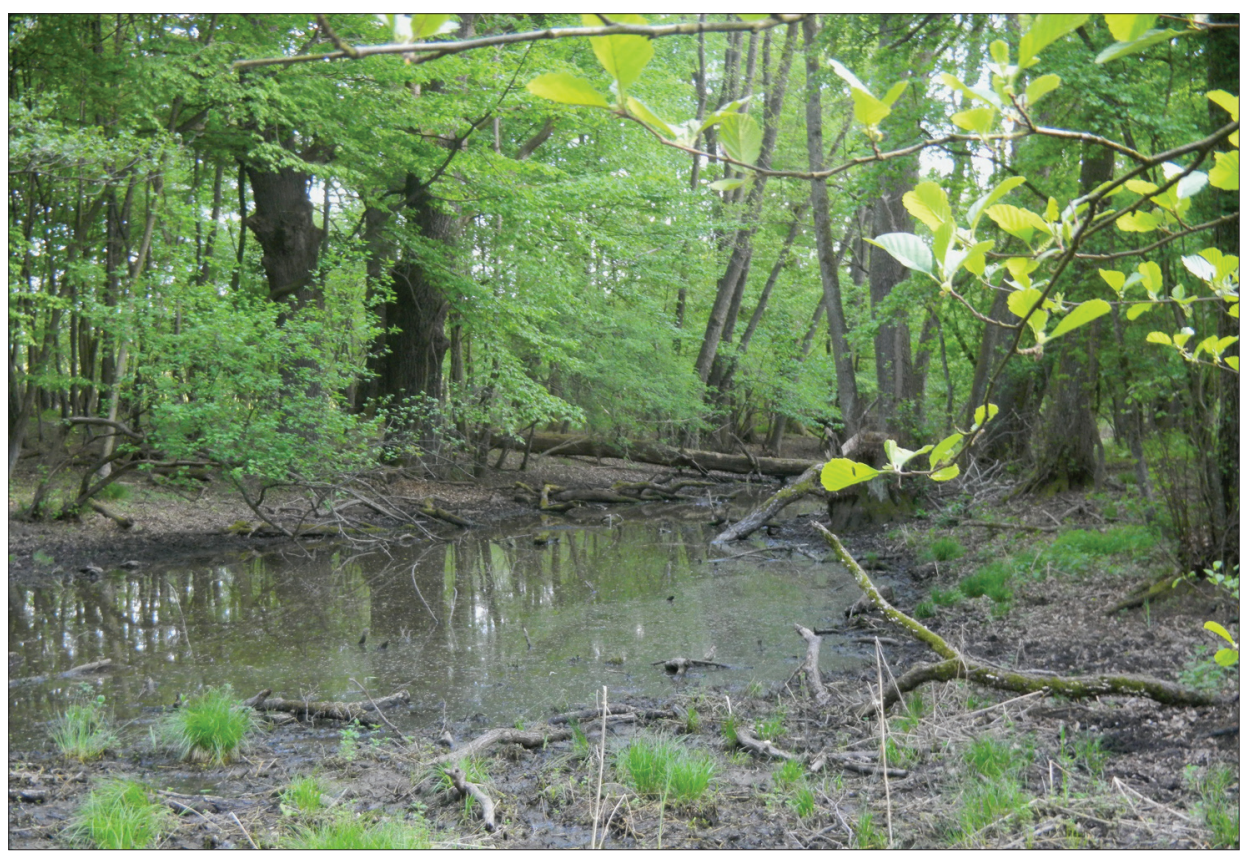

Fig. 6: Swamp at Csokonyavisonta Wooded Pasture 


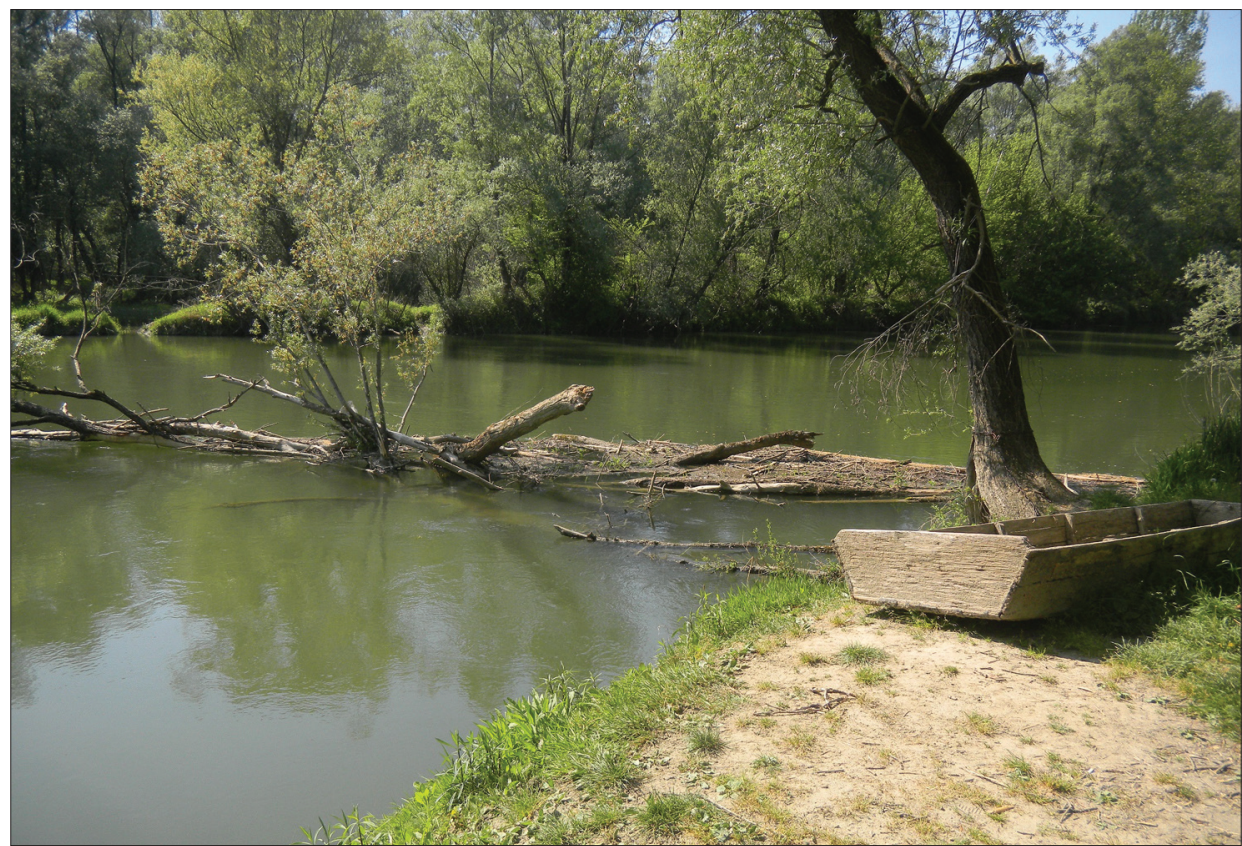

Fig. 7: The River Dráva at Vízvár

Dolerus (Poodolerus) stygius Förster, 1860 (=Dolerus megapterus sensu Zombori, 1982): Csokonyavisonta: Protected Wooded Pasture, 10. 04. 2020, 1 female. Rare. Larva on Carex spp.

Dolerus (Achaetoprion) triplicatus (Klug, 1818): Csokonyavisonta: Protected Wooded Pasture, 10. 04. 2020, 1 male. Sporadic. Larva on Juncus filiformis and J. effusus.

Dolerus (Dicrodolerus) vestigialis (Klug, 1818): Csokonyavisonta: Protected Wooded Pasture, 23. 04. 2020, 1 male, 04. 05. 2020, 1 male. Common. Host plants: Equisetum palustre, E. sylvaticum, E. arvense and E. pratense.

\section{Selandrinae}

Birka (Birka) cinereipes (Klug, 1816): Vízvár: Riverside, 24. 04. 2020, 1 male; Csokonyavisonta: Protected Wooded Pasture, 23. 05. 2020, 1 male. Sporadic. Host plants: Myosotis spp.

Nesoselandria morio (Fabricius, 1781): Csokonyavisonta: Protected Wooded Pasture, 23. 05. 2020, 3 females, 1 male, 16. 05. 2020, 3 males, 03. 05. 2020, 1 male. Frequent. Host plants: Brachytecium reflexum, Ceratodon purpureus, Chenopodium album, Dicranum scoparium, Fragaria vesca, Hedwigia ciliata, Myosotis arvensis, Plagiomnium cuspidatum, Plagiothecium denticulatum, Polygonum aviculare, Polytrichum commune, Pseudobryum cinclidiodes, Sanionia uncinata, Stellaria media, Veronica chamaedrys and V. officinalis.

Selandria serva (Fabricius, 1793): Csokonyavisonta: Protected Wooded Pasture, 09. 05. 2020, 1 female. Frequent. Host plants: Poaceae, Carex spp. and Juncus spp. 


\section{Allantinae}

Allantus (Emphytus) calceatus (Klug, 1818): Csokonyavisonta: Protected Wooded Pasture, 11. 04. 2020, 1 female, 1 male, 18. 04. 2020, 2 females, 2 males, 16. 04. 2020, 2 females, 19. 04. 2020, 1 female, 10. 04. 2020, 2 males. Generally sporadic, here frequent. Host plants: Rubus, Sanguisorba, Rosa, Filipendula, Fragaria and Alchemilla spp.

Ametastegia (Ametastegia) equiseti (Fallén, 1808): Csokonyavisonta: Protected Wooded Pasture, 03. 05. 2020, 1 female, 09. 05. 1 male. Frequent. Larva on Chenopodium album, Lythrum salicaria, Polygonum persicaria and Rumex acetosella.

Ametastegia (Ametastegia) glabrata (Fallén, 1808): Csokonyavisonta: Protected Wooded Pasture, 26. 04. 2020, 1 female, 17. 04. 2020, 1 female. Frequent. Larva on Chenopodiaceae, Polygonaceae, Plantago, Salix, Lithrum, Ribes and Rubus spp.

Ametastegia (Protemphytus) carpini (Hartig, 1837): Csokonyavisonta: Protected Wooded Pasture, 22. 04. 2020, 1 female. Frequent. Host plant: Geranium spp.

Ametastegia (Protemphytus) tenera (Fallén, 1808): Csokonyavisonta: Protected Wooded Pasture, 10. 05. 2020, 2 males, 11. 05. 2020, 1 male, 24. 04. 2020, 1 male. Frequent. Larva on Rumex spp.

Athalia bicolor Serville, 1823: Babócsa: Két-árok köze, 09. 05. 2020, 1 female, 1 male, 23. 05. 2020, 2 females, 23. 05. 2020, 3 males, 02. 05. 2020, 1 male; Csokonyavisonta: Protected Wooded Pasture, 10. 05. 2020, 1 female, 30. 05. 2020, 3 males, 09. 05. 2020, 3 males, 11. 05. 2020, 1 male; Darány: Kis-tó, 26. 04. 2020, 2 females. Frequent. Host plant: Ranunculus spp.

Athalia cordata Serville, 1823: Csokonyavisonta: Protected Wooded Pasture, 10. 05. 2020, 1 female, 21. 04. 2020, 1 female, 2 males, 11. 05. 2020, 1 female, 16. 05. 2020, 1 female, 17. 04. 2020, 1 male, 16. 04. 2020, 1 male, 19. 04. 2020, 1 male, 18. 04. 2020, 1 male, 23. 04. 2020, 3 males, 11. 04. 2020, 1 male, 23. 05. 2020, 1 male, 04. 05. 2020, 2 males; Darány: Kis-tó, 16. 04. 2020, 2 females, 22. 04. 2020, 1 female, 18. 04. 2020, 1 male, 26. 04. 2020, 3 males; Vízvár: Riverside, 19. 04. 2020, 1 female. Common. Larva on Misopates orontinum, Antirrhinum majus, Ajuga reptans, Teucrium scorodonia and Plantago spp.

Athalia lugens (Klug, 1815): Vízvár: Riverside, 09. 05. 2020, 1 male. Sporadic. Feeding on various Cruciferae.

Athalia circularis (Klug, 1815): Csokonyavisonta: Protected Wooded Pasture, 11. 05. 2020, 1 female, 23. 04. 2020, 1 male, 02. 05. 2020, 1 male, 04. 05. 2020.1 male; Vízvár: Riverside, 24. 04. 2020, 1 male. Frequent. Host plants: Arctium lappa, Ajuga reptans, Veronica beccabunga, V. longifolia, V. officinalis, Alliaria petiolata, Glechoma hederacea, Melampyrum, Capsella and Lycopus spp.

Athalia rosae (Linné, 1758): Csokonyavisonta: Protected Wooded Pasture, 21. 04. 2020, 1 female, Vízvár: Riverside, 24. 04. 2020, 1 female; Bolhó: Libalegelő, 19. 07. 2020, 48 males, 24 females, 26. 07. 2020, 4 females, 1 male, 02. 08. 2020, 3 males, 2 females; Darány: Kis-tó, 19. 07. 2020, 1 female; Common pest. Host plants: Raphanus sativus, $R$. raphanistrum, Sinapis arvensis, Sisymbrium officinale, Armoracia rusticana, Barbarea sp., Brassica napus, B. juncea, B. rapa, B. oleracea, Tropaeolum majus, Sinapis arvensis, Alliara petiolata and Cardamine spp.

Empria sexpunctata (Serville, 1823): Csokonyavisonta: Protected Wooded Pasture, 03. 05. 2020, 2 females. Frequent. Larva on Geum spp.

Empria liturata (Gmelin, 1790): Csokonyavisonta: Protected Wooded Pasture, 11. 04. 2020, 2 females, 12. 04. 2020, 3 females, 1 male, 16. 04. 2020, 1 female, 1 male, 17. 04. 2020, 2 females, 18. 04. 2020, 2 females, 2 males, 19. 04. 2020, 2 females, 3 males, 22. 04. 2020, 1 female, 26. 04. 2020, 1 female, 26. 04. 2020, 1 female, 19. 04. 2020, 1 
female, 04. 05. 2020, 1 female, 09. 04. 2020, 1 male, 21. 04. 2020, 1 male, 09. 05. 2020, 1 male; Vízvár: Riverside, 24. 04. 2020, 1 female. Frequent. Host plants: Fragaria and Geum spp.

Eriocampa ovata ssp. ovata (Linné, 1760): Babócsa: Két-árok köze, 16. 05. 2020, 1 female, 23. 05. 2020, 1 female; Csokonyavisonta: Protected Wooded Pasture, 23. 05. 2020, 3 females. Frequent. Larva on Alnus.

Eriocampa umbratica (Klug, 1816): Csokonyavisonta: Protected Wooded Pasture, 23. 04. 2020, 1 female, 04. 05. 2020, 1 female, 1 male, 09. 05. 2020, 1 female, 02. 05. 2020, 2 males, 24. 04. 2020, 1 male, 22. 04. 2020, 1 male, 23. 04. 2020, 1 male, 16. 05. 2020, 1 male; Vízvár: Riverside, 24. 04. 2020, 1 female. Frequent. Larva on Alnus.

Harpiphorus lepidus (Klug, 1818): Csokonyavisonta: Protected Wooded Pasture, 03. 05. 2020, 1 female. Sporadic. Larva on Quercus.

Monsoma pulveratum (Retzius, 1783): Csokonyavisonta: Protected Wooded Pasture, 03. 05. 2020, 1 female. Sporadic. Larva on Alnus spp.

Taxonus agrorum (Fallén, 1808): Csokonyavisonta: Protected Wooded Pasture, 21. 04. 2020, 1 female, 23. 04. 2020, 1 female; Vízvár: Riverside, 23. 05. 2020, 1 male. Frequent. Host plants: Rubus idaeus and R. caesius.

\section{Heterarthrinae}

Caliroa cothurnata (Serville, 1823): Csokonyavisonta: Protected Wooded Pasture, 11. 05. 2020, 1 female. New record for Hungary. Larva on Quercus spp.

\section{Blennocampinae}

Blennocampa phyllocolpa Viitasaari \& Vikberg, 1985: Csokonyavisonta: Protected Wooded Pasture, 03. 05. 2020, 1 female. Frequent. Larva rolls the leaves of Rosa spp.

Cladardis elongatula (Klug, 1817): Csokonyavisonta: Protected Wooded Pasture, 03. 05. 2020, 1 female. Sporadic. Larva bores in shoots of Rosa spp.

Claremontia alternipes (Klug, 1816): Csokonyavisonta: Protected Wooded Pasture, 23. 04. 2020, 1 female; Vízvár: Riverside, 24. 04. 2020, 1 female. Sporadic. Host plant: Rubus idaeus.

Claremontia brevicornis (Brischke, 1883): Csokonyavisonta: Protected Wooded Pasture, 26. 04. 2020, 1 female. Frequent. Host plants: Fragaria spp., Sanguisorba spp. and Potentilla reptans.

Claremontia waldheimii (Gimmerthal, 1847): Csokonyavisonta: Protected Wooded Pasture, 21. 04. 2020, 1 female, 17. 04. 2020, 1 female. Frequent. Host plant: Geum urbanum.

Eutomostethus ephippium (Panzer, 1798): Csokonyavisonta: Protected Wooded Pasture, 11. 05. 2020, 1 female, 09. 05. 2020, 1 female, 4 males, 02. 05. 2020, 1 female, 2 males, 24. 04. 2020, 5 males, 10. 05. 2020, 2 males, 10. 05. 2020, 1 male, 11. 05. 2020, 8 males, 16. 05. 2020, 5 males, 23. 05. 2020, 5 males, 04. 05. 2020, 1 male, 03. 05. 2020, 1 male, 04. 05. 2020, 4 males; Vízvár: Riverside, 24. 04. 2020, 1 female, 19. 04. 2020, 2 males, 24. 04. 2020, 1 male; Darány: Kis-tó, 22. 04. 2020, 2 males, 03. 05. 2020, 1 male. Common, larva on Poaceae.

Eutomostethus gagathinus (Klug, 1816): Csokonyavisonta: Protected Wooded Pasture, 11. 05. 2020, 2 females, 10. 05. 2020, 1 female, 04. 05. 2020, 2 females, 09. 05. 2020, 1 male. Frequent. Host plant: Carex paniculata.

Eutomostethus luteiventris (Klug, 1816): Csokonyavisonta: Protected Wooded Pasture, 09. 04. 2020, 1 female, 10. 04. 2020, 3 females, 11. 04. 2020, 5 females, 12. 04. 2020, 4 females, 16. 04. 2020, 1 female, 17. 04. 2020, 2 females, 18. 04. 2020, 2 females, 21. 04. 2020, 2 females, 22. 04. 2020, 2 females, 03. 05. 2020, 1 female, 04. 05. 2020, 2 females, 


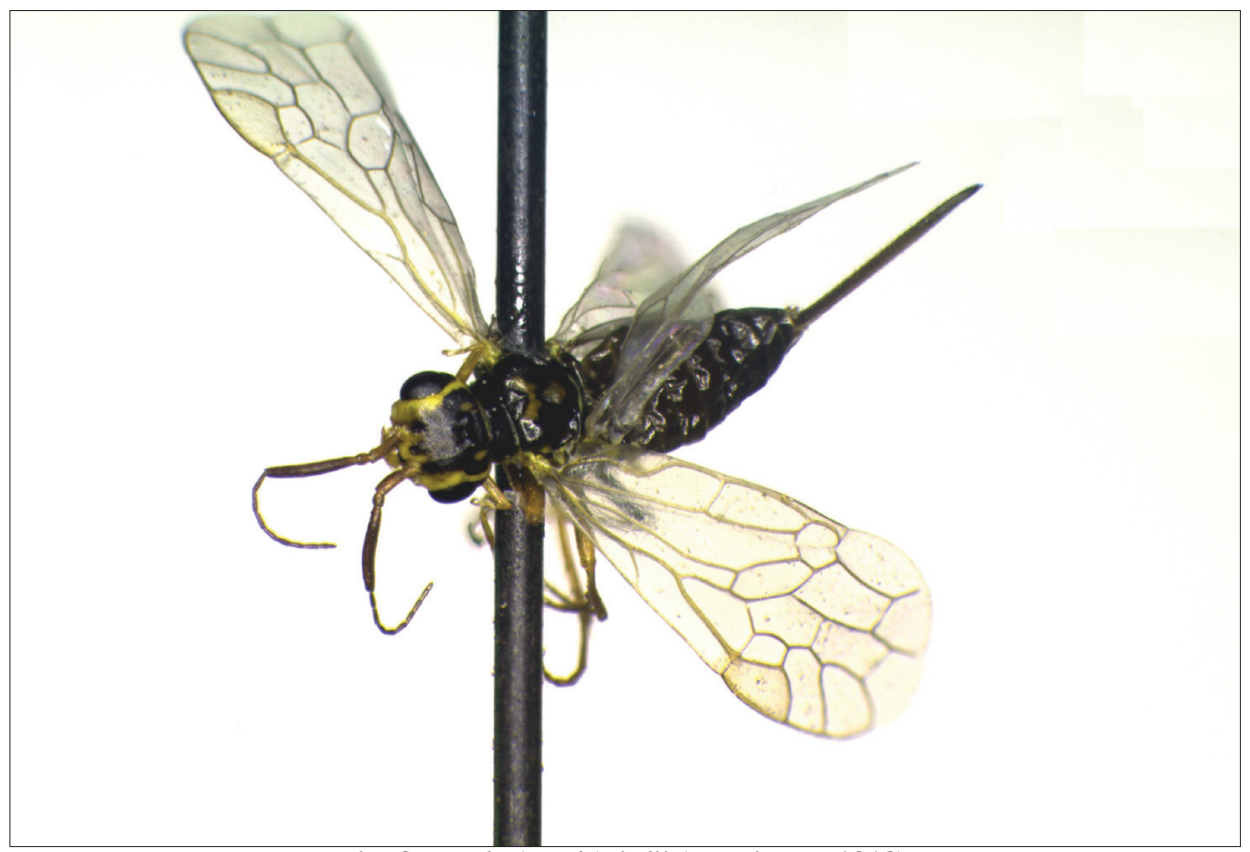

Fig. 8: Xyela (Xyela) julii (Brébisson, 1818)

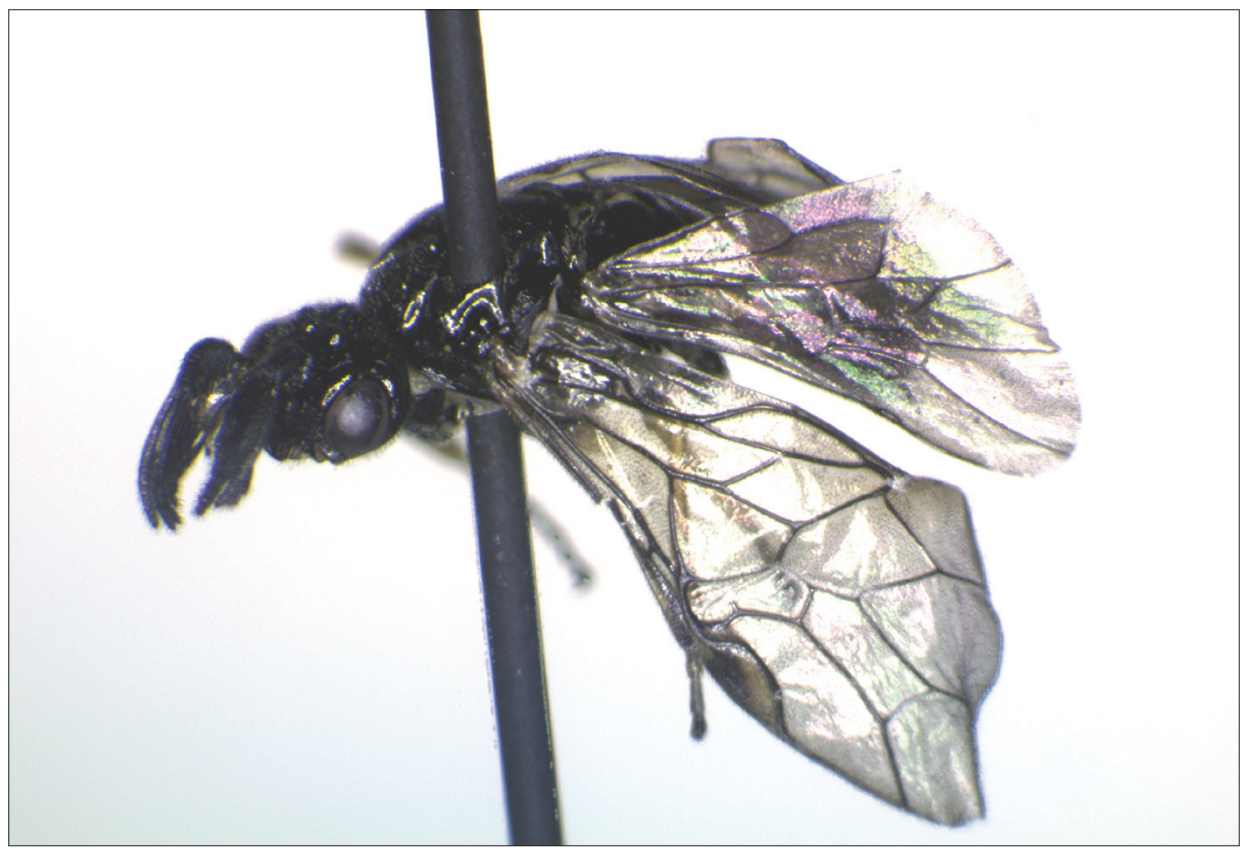

Fig. 9: Monoctenus juniperi (Linné, 1758) 
09. 05. 2020, 2 females; Vízvár: Riverside, 24. 04. 2020, 1 female. Frequent, locally common. Larva on Juncus effusus.

Eutomostethus punctatus (Konow, 1887): Csokonyavisonta: Protected Wooded Pasture, 10. 05. 2020, 1 female, 02. 05. 2020, 8 males, 03. 05. 2020, 1 male, 19. 04. 2020, 1 male, 23. 04. 2020, 1 male; Vízvár: Riverside, 09. 05. 2020, 1 male, 24. 04. 2020, 2 males. Sporadic. Locally frequent. Only with few localities. Host plant: Carex paniculata.

Monophadnus pallescens (Gmelin, 1790): Csokonyavisonta: Protected Wooded Pasture, 11. 04. 2020, 1 female, 12. 04. 2020, 6 females, 18. 04. 2020, 1 female, 02. 05. 2020, 1 female, 10. 05. 2020, 1 female. Common. Host plants: Ranunculus acris, $R$. repens, $R$. lanuginosus and Anemone nemorosa.

Monophadnus monticola (Hartig, 1837): Csokonyavisonta: Protected Wooded Pasture, 10. 04. 2020, 1 female, 16. 04. 2020, 1 female, 18. 04. 2020, 1 female; Darány: Kis-tó, 16. 04. 2020, 1 female; Vízvár: Riverside, 24. 04. 2020, 1 female. Sporadic. Larva on Helleborus spp.

Stethomostus fuliginosus (Schrank, 1781): Csokonyavisonta: Protected Wooded Pasture, 24. 04. 2020, 1 male, 02. 05. 2020, 1 male. Frequent. Larva on Ranunculus acris, $R$. repens and $R$. sceleratus.

\section{Tenthredininae}

Aglaostigma (Astochus) aucupariae (Klug, 1817): Csokonyavisonta: Protected Wooded Pasture, 23. 04. 2020, 1 female, 26. 04. 2020, 1 female, 11. 04. 2020, 2 males, 03. 05. 2020, 1 male. Common. Larva on Galium mollugo and G. boreale.

Aglaostigma (Astochus) fulvipes (Scopoli, 1763): Csokonyavisonta: Protected Wooded Pasture, 10. 04. 2020, 1 male, 17. 04. 2020, 6 males, 23. 04. 2020, 1 male, 18. 04. 2020, 1 male, 21. 04. 2020, 1 male, 11. 04. 2020, 1 female, 24. 04. 2020, 2 females, 12. 04. 2020, 2 females, 16. 04. 2020, 1 female, 03. 05. 2020, 1 female; Vízvár: Riverside, 19. 04. 2020, 2 males, 19. 04. 2020, 1 female; Babócsa: Két-árok köze, 02. 05. 2020, 1 female. Common. Larva on Galium mollugo and G. verum.

Macrophya (Macrophya) albicincta (Schrank, 1776): Csokonyavisonta: Protected Wooded Pasture, 02. 05. 2020, 1 female, 03. 05. 2020, 1 female, 04. 05. 2020, 1 female, 10. 05. 2020, 1 female, 16. 04. 2020, 1 female, 17. 04. 2020, 1 female, 19. 04. 2020, 1 female, 22. 04. 2020, 2 females, 23. 04. 2020, 3 females, 24. 04. 2020, 1 female, 1 male, 26. 04. 2020, 1 female, 21. 04. 2020, 2 males; Vízvár: Riverside, 24. 04. 2020, 1 female, 3 males; Darány: Kis-tó, 22. 04. 2020, 1 male, 23. 04. 2020, 2 males. Common. Host plants: Sambucus ebulus, S. nigra, S. racemosa, Valeriana officinalis and Viburnum opalus.

Macrophya (Macrophya) annulata (Geoffroy, 1785): Csokonyavisonta: Protected Wooded Pasture, 16. 05. 2020, 1 female, 23. 05. 2020, 7 females, 2 males, 09. 05. 2020, 3 females, 02. 05. 2020, 1 male, 10. 05. 2020, 2 males, 11. 05. 2020, 1 male. Frequent. Larva on Potentilla reptans, Origanum vulgare, Euphorbia, Rosa, Rubus and Sambucus spp.

Macrophya (Macrophya) duodecimpunctata (Linné, 1758): Babócsa: Két-árok köze, 16. 05. 2020, 1 female; Csokonyavisonta: Protected Wooded Pasture, 09. 05. 2020, 1 male, 10. 05. 2020, 1 male. Frequent. Host plants: Graminae, Cyperaceae and Carex spp.

Macrophya (Macrophya) montana ssp. montana (Scopoli, 1763): Csokonyavisonta: Protected Wooded Pasture, 04. 05. 2020, 1 female, 1 male, 09. 05. 2020, 1 female, 02. 05. 2020, 2 males, 10. 05. 2020, 2 males; Vízvár: Riverside, 23. 05. 2020, 1 female; Babócsa: Két-árok köze, 23. 05. 2020, 1 female, 09. 05. 2020, 1 male. Common. Host plant: Rubus caesius. 


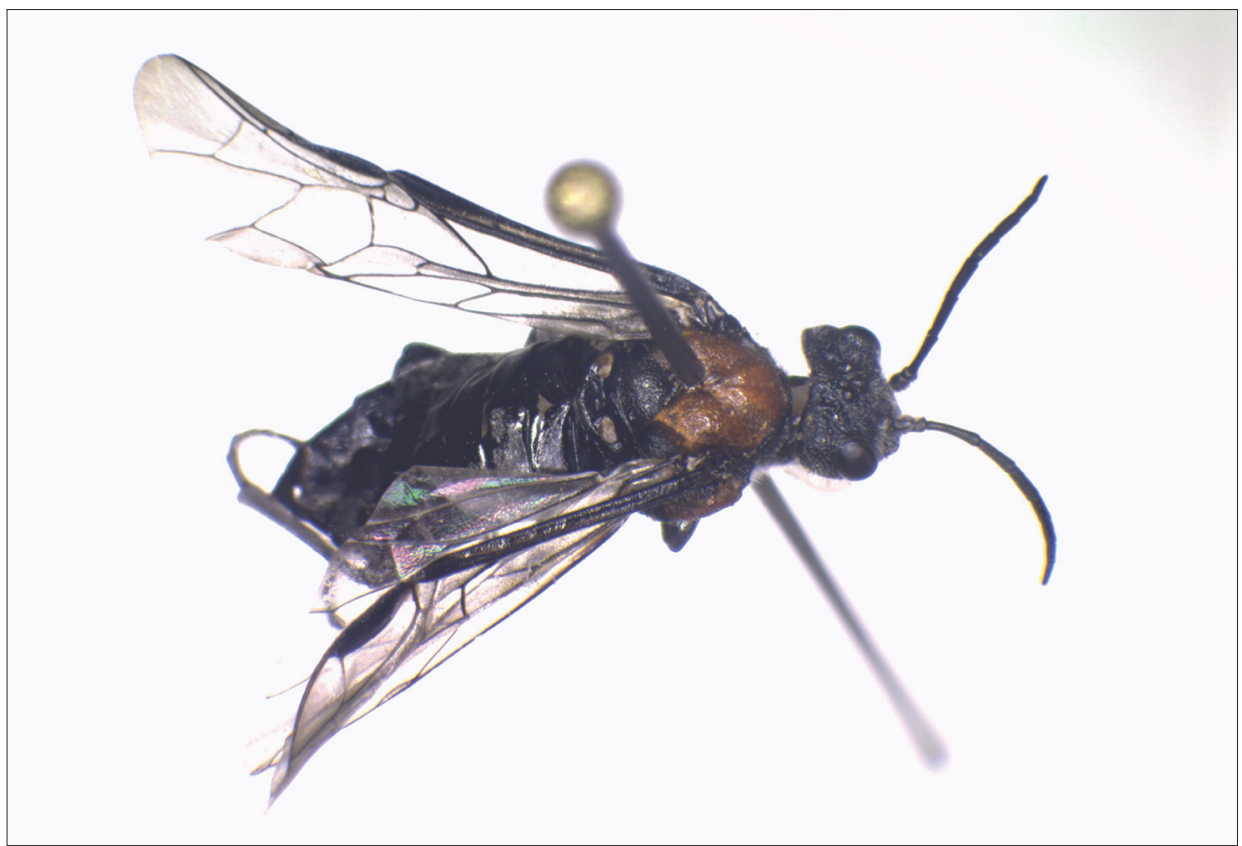

Fig. 10: Dolerus (Poodolerus) blanki Liston, 1995

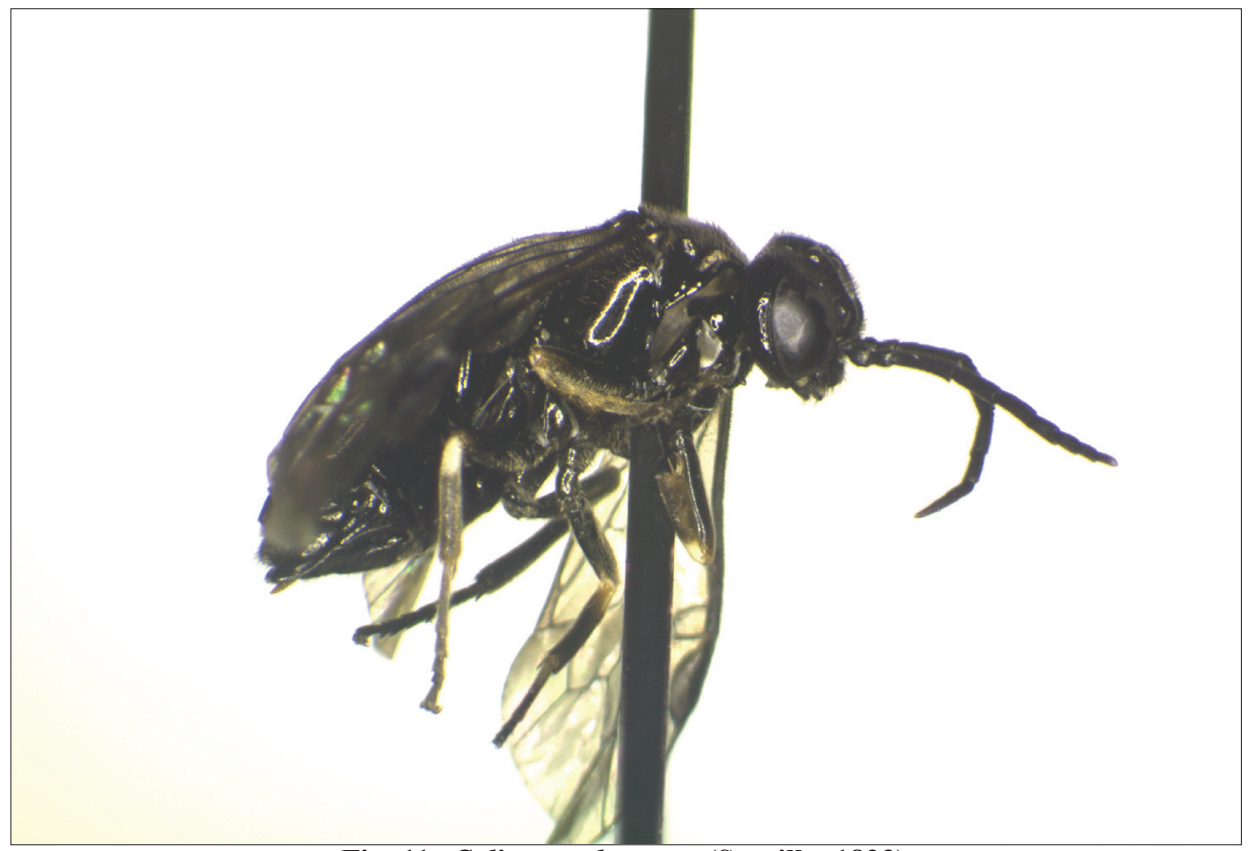

Fig. 11: Caliroa cothurnata (Serville, 1823) 
Macrophya (Macrophya) sanguinolenta (Gmelin, 1790): Csokonyavisonta: Protected Wooded Pasture, 09. 05. 2020, 1 male, 2 females, 16. 05. 2020, 1 male, 23. 05. 2020, 1 male, 03. 05. 2020, 1 female. Generally sporadic, here frequent. Larva on Galeopsis spp., Senecio spp. and Veronica spp.

Sciapteryx consobrina (Klug, 1816): Csokonyavisonta: Protected Wooded Pasture, 11. 04. 2020, 1 female, 12. 04. 2020, 1 female, 24. 04. 2020, 1 female; Darány: Kis-tó, 18. 04. 2020, 2 females. Frequent. Larval hosts: Adoxa spp., Anemone spp., Ranunculus acris and Ranunculus ficaria.

Perineura rubi (Panzer, 1803): Csokonyavisonta: Protected Wooded Pasture, 16. 04. 2020, 1 female. 02. 05. 2020, 1 female. Sporadic. Host plant unknown. Adults associated with Rubus spp.

Pachyprotasis rapae (Linné, 1767): Csokonyavisonta: Protected Wooded Pasture, 23. 04. 2020, 1 female, 26. 04. 2020, 1 female, 04. 05. 2020, 1 female, 09. 05. 2020, 1 female, 10. 05. 2020, 1 male, 11. 05. 2020, 1 male, 03. 05. 2020, 1 male, 02. 05. 2020, 1 male. Frequent. Host plants: Solanum tuberosum, Pedicularis palustris, Angelica sylvestris, Veronica beccabunga, Betonica officinalis, Corylus avellana, Salix caprea, Fraxinus excelsior, Tussilago farfara, Symphoricarpos albus, Scrophularia, Solidago, Verbascum, Origanum, Atropa, Sarothamnus, Senecio, Polygonum, Aspidium, Epilobium, Hypericum, Galeopsis, Mentha, Polystichum, Plantago, Quercus and Stachys spp.

Tenthredopsis friesei (Konow, 1884): Csokonyavisonta: Protected Wooded Pasture, 16. 05. 2020, 1 female, 30. 05. 2020, 1 female. Frequent. Host plants: Holcus mollis and other Poaceae.

Tenthredopsis litterata (Geoffroy, 1785): Csokonyavisonta: Protected Wooded Pasture, 09. 05. 2020, 1 male, 16. 05. 2020, 2 males. Frequent. Larva on Agrostis, Dactylis and Calamagrostis spp.

Tenthredopsis nassata (Linné, 1767): Babócsa: Két-árok köze, 09. 05. 2020, 1 female. Earlier frequent, recently sporadic. Host plants: Dactylis glomerata, Deschampsia caespitosa, D. calmagrostis, Flexuosa spp., Holcus spp., Lolium perenne, Agropyron spp., Carex spp., Anthriscus silvestris and Artemisia spp.

Tenthredopsis ornata (Serville, 1823): Csokonyavisonta: Protected Wooded Pasture, 16. 05. 2020, 2 females. Frequent. Larva on Brachypodium sylvaticum.

Tenthredopsis sordida (Klug, 1817): Csokonyavisonta: Protected Wooded Pasture, 02. 05. 2020, 6 males, 04. 05. 2020, 3 males, 03. 05. 2020, 5 males, 1 female, 11. 05. 2020, 2 males, 23. 04. 2020, 1 male; Babócsa: Két-árok köze, 09. 05. 2020, 1 female. Frequent. Larva on Arrhenatherum elatius, Lolium perene, Carex spp., Calamagrostis spp. and Dactylis glomerata.

Tenthredopsis stigma (Fabricius, 1798): Csokonyavisonta: Protected Wooded Pasture, 10. 05. 2020, 1 female. Frequent. Known host plant: Triticum intermedium.

Tenthredopsis tessellata (Klug, 1817): Csokonyavisonta: Protected Wooded Pasture, 22. 04. 2020, 1 female, 24. 04. 2020, 1 female. Sporadic. Larva on Deschampsia, Dactylis, Aira and Lolium spp.

Tenthredo (Tenthredella) atra Linné, 1758: Csokonyavisonta: Protected Wooded Pasture, 10. 05. 2020, 1 male. Frequent. Larval hosts: Lamium, Mentha, Plantago, Vicia, Ranunculus, Scabiosa, Brassica and Solanum spp.

Tenthredo (Tenthredo) zona Klug, 1817: Csokonyavisonta: Protected Wooded Pasture, 11. 04. 2020, 1 female. Sporadic. Host plant: Hypericum perforatum.

Tenthredo (Zonuledo) distinguenda (Stein, 1885): Csokonyavisonta: Protected Wooded Pasture, 04. 05. 2020, 1 female, 1 male, 09. 05. 2020, 1 male. Frequent. Host plant unknown. 
Tenthredo (Temuledo) temula Scopoli, 1763: Csokonyavisonta: Protected Wooded Pasture, 21. 04. 2020, 1 female. Frequent, locally common. Larva on Ligustrum and Origanum spp.

\section{Nematinae}

Cladius (Cladius) pectinicornis (Geoffroy, 1785): Csokonyavisonta: Protected Wooded Pasture, 16. 05. 2020, 1 male. Common. Host plant: Rubus spp.

Euura fuscomaculata (Förster, 1854) (=Nematus fuscomaculatus Förster, 1854): Csokonyavisonta: Protected Wooded Pasture, 22. 04. 2020, 1 female, 23. 04. 2020, 1 female. Rare. It was recorded from Populus tremula.

Euura leucosticta (Hartig, 1837) (=Phyllocolpa leucosticta (Hartig, 1837): Csokonyavisonta: Protected Wooded Pasture, 16. 05. 2020, 1 female, 03. 05. 2020, 1 female; Darány: Kis-tó, 03. 05. 2020, 1 female. Frequent. Larva on Salix capreae, $S$. aurita, S. atrocinerea and $S$. cinerea.

Euura mucronata (Hartig, 1837): Csokonyavisonta: Protected Wooded Pasture, 03. 05. 2020, 1 male. Host plants: Salix spp. Rare.

Euura myosotidis (Fabricius, 1804) (=Nematus myosotidis (Fabricius, 1804): Csokonyavisonta: Protected Wooded Pasture, 18. 04. 2020, 1 female, 16. 04. 2020, 1 female, 16. 05. 2020, 1 female, 03. 05. 2020, 1 male, 02. 05. 2020, 1 male, 09. 05. 2020, 1 male, 10. 05. 2020, 1 male, 11. 05. 2020, 1 male, 23. 05. 2020, 1 male, 16. 05. 2020, 1 male; Babócsa: Két-árok köze, 09. 05. 2020, 1 male. Common. Larval hosts: Onobrychis and Trifolium spp.

Euura vaga (Fabricius, 1781) (=Pachynematus vagus (Fabricius, 1781)): Csokonyavisonta: Protected Wooded Pasture, 04. 05. 2020, 1 female, 26. 04. 2020, 1 female, 11. 05. 2020, 1 male. Larva on Carex spp. Frequent.

Hoplocampa fulvicornis (Panzer, 1801): Drávatamási, Old Railway 10. 04. 2020, 1 female. Sporadic. Larva on Prunus spinosa.

Nematinus luteus (Panzer, 1803): Csokonyavisonta: Protected Wooded Pasture, 21. 04. 2020, 1 female. Sporadic. Larva on Alnus spp.

Nematus lucidus (Panzer, 1801): Csokonyavisonta: Protected Wooded Pasture, 19. 04. 2020, 1 female, 09. 05. 2020, 1 female. Frequent. Larva on Crataegus and Prunus spinosa.

Platycapus luridiventris (Fallén, 1808): Csokonyavisonta: Protected Wooded Pasture, 03. 05. 2020, 1 male. Larva on Alnus glutionosa and A. incana. Sporadic.

Pristiphora armata (Thomson, 1863): Csokonyavisonta: Protected Wooded Pasture, 11. 05. 2020, 1 female, 04. 05. 2020, 1 female, 22. 04. 2020, 1 female, 3 males, 17. 04. 2020, 1 male, 16. 04. 2020, 2 males, 19. 04. 2020, 2 males, 18. 04. 2020, 4 males, 21. 04. 2020, 7 males, 23. 04. 2020, 2 males, 24. 04. 2020, 1 male, 03. 05. 2020, 1 male, 02. 05. 2020, 4 males; Vízvár: Riverside, 24. 04. 2020, 1 female, 1 male; Babócsa: Két-árok köze, 16. 05. 2020, 2 males. Frequent. Larva on Crataegus spp.

Pristiphora pallidiventris (Fallén, 1808): Csokonyavisonta: Protected Wooded Pasture, 16. 04. 2020, 1 female, 22. 04. 2020, 1 female, 11. 04. 2020, 1 male. Frequent. Larva on Geum, Potentilla, Rubus and Filipendula spp. Filipendula ulmaria, Geum urbanum, G. rivale, Rubus chamaemorus, $R$. idaeus, $R$. fruticosus and $R$. ulmifolius. 


\section{Results}

100 species of 683 specimens were collected in the Duna-Dráva National Park and the adjecent territory: Csokonyavisonta Wooded Pasture. According to Zombori, 1985: "In the last 10 years, 143 specimens were collected, representing 45 species". I can confirm, the observation of Zombori. The species richness and population density of sawflies are very low in the Dráva region. Here only $29 \%$ of the specimens were collected, and only 41 species.

The majority of the specimens, exemplars were collected significantly north of the Dráva Region, in Csokonyavisonta Protected Wooded Pasture (482 specimens). This small area gave $70.6 \%$ of the total collected material. From the 100 species collected in the region, 59 species were captured only in Csokonyavisonta Protected Wooded Pasture.

The dominant species was Athalia rosae (Linné, 1758) with 85 exemplars. Other frequent species (with 20 or more collected exemplars) were Eutomostethus ephippium (Panzer, 1798), Dolerus (Poodolerus) nigratus (O.F. Müller, 1776), Athalia cordata Serville, 1823, Empria liturata (Gmelin, 1790), Macrophya (Macrophya) albicincta (Schrank, 1776), Aglaostigma (Astochus) fulvipes (Scopoli, 1763), Eutomostethus luteiventris (Klug, 1816), Eutomostethus ephippium (Panzer, 1798) Pristiphora armata (Thomson, 1863) and Arge cyanocrocea (Forster, 1771). These 11 species (with 342 exemplars) amounts up to $50 \%$ of the total collected material.

\section{Rare and interesting species}

Xyela (Xyela) julii (Brébisson, 1818): In the Carpathian Basin, this species is sporadic but extremely rare in Hungary. We have only one historical exemplare from Törökbálint. This is the second specimen from Hungary. Fig. 8.

Aproceros leucopoda Takeuchi, 1939: Recently invaded Europe, known from Hungary, Poland, Slovakia, Austria, Romania, Ukraine and the Russian Far East (BLANK et al. 2010, CsóKA et al. 2010).

Monoctenus juniperi (Linné, 1758): Widely distributed, sporadic in the Carpathian Basin but rare in Hungary. We have records from Visegrád, Bakony, Pilis, Csepel, Csévharaszt and Piliscsaba. From the Juniper Woodland at Darány, only the other species, Monoctenus obscuratus (Hartig, 1837) was recorded. Fig. 9.

Dolerus (Poodolerus) blanki Liston, 1995: From the following sites, we have records from the Carpathian Basin: from Slovakia: Humenné (Homonna), from Hungary: Szeged, Fehértó, Kaposvár: Tókaji parkerdő, Kis Balaton: Zala part, Darány, from Transylvania: Hátszeg (Hateg), Tasnád (Tasnad), Peér (Pir), Nagyszeben (Sibiu), Magura, Vízakna (Ocna Sibiului), Szenterzsébet (Gusterita), Pöltinis (Paltinis), Gyilkos tó (Lacu Rosu), Fogaras (Mt. Fagaras), Szinája (Sinaia) and from Coratia: Ogulin. Rare, widely distributed European species. Fig. 10.

Caliroa cothurnata (Serville, 1823): New record for Hungary and for the Carpathian Basin. It is a recently revised species (LACOURT 2002), so far it has been considered as synonym of Caliroa cinxia (Klug, 1816). Differences of the 2 species: Caliroa cothurnata (Serville, 1823): The spot on hind tibia is clearly marked with white: in the male, between the fifth and the basal quarter of the tibia is clearly white; in the female, between one third and one quarter of the base of hind tibia is clearly white. Head with short hairs. On anterior wings, the smoky transverse bland is clearly visible, this spot is delimited by the 1 st recurrent vein and the oblique apical part of the median vein. Posterior wings of females are always with two closed median cells. In Caliroa cinxia (Klug, 1816): the white spot on hind tibia smaller and weaker: in the male, only the knee 
with small white spot and a light brown trail which is more or less developed along the tibia; in the female, a conspicuous white spot occupies the basal fifth of the hind tibia. Head with long hairs. The diffuse smoky band of anterior wing is poorly delimited laterally. Hind wings of females with two closed median cells in 30 to $40 \%$ of cases or with a single closed median cell in 60 to $70 \%$ of the individuals. It has been recorded from Germany, Italy, Finland, Poland, France and Luxembourg. Fig. 11.

Euura fuscomaculata (Förster, 1854) (=Nematus fuscomaculatus Förster, 1854): From Hungary it is only known from Tákos. Other places of capture from the Carpathian Basin: from Slovakia: Kňaží vrch (Pap kő), Vranov (Varannó), Viničky (Szőllőske), Tatranská Lomnica (Tátralomnic), from Romania: Poiana-Teiului (Piatra Nemat), Szeben (Sibiu), Szucsáva (Suceava), Moldova and Magura (Magura Cisnadiei) From Croaqtia: Kopacko jezero, Hordovanj, from Bosnia: Ildze and from the Czech part of Tatras: Trojačka and Jičina. Widely distributed, rare European species.

\section{Species diversity and endangering factors on the main sampling sites}

\section{Csokonyavisonta}

The protected wooded pasture at Csokonyavisonta village holds the highest diversity of sawflies with numerous rare species and divers habitats. Unfortunately, the traditional grazing and sheep farming was completely abandoned and the vegetation of the pasture isn't cut. Therefore, the shrubby pioneer vegetation occupies more and more territories year by year. The main endangering factors for the pasture are the invasive daisy fleabane (Stenactis annua L.) in dry spots and goldenrode (Solidago sp.) in wet spots. Black cherry (Prunus serotina Ehrh.) and common milkweed (Asclepias syriaca L.) are also frequent.

\section{Babócsa}

Along the Rinya brook, the pasture is regularly cut, however it is still far not enough to prevent the spreading of fleabane (Stenactis annua L.) and goldenrode (Solidago sp.), the dominant invasive species of this sampling site. Population density and species richness of sawflies are low. Black cherry (Prunus serotina Ehrh.) and common milkweed (Asclepias syriaca L.) are also present.

\section{Vízvár}

The sampling site at Vízvár is willow dominated floodplain forest with wet meadow spots. The sawfly density and species richness is low. The most frequent invasive plants are goldenrode (Solidago sp.) and black locust (Robinia pseudoacacia L.). Common ragweed (Ambrosia artemisiifolia L.) is also frequent.

\section{Darány: Juniper woodlands}

Very special habitats, unique in Hungary probably even in the Carpathian Basin. Since it is dominated by Juniper trees on sand dunes, the sawfly biodiversity is extremely low (only 3 species, 2 of them were captured this year) but very special and valuable. The sand dunes are endangered by black locust (Robinia pseudoacacia L.) and in the forested parts by black cherry (Prunus serotina Ehrh.) is dominated. Ailanthus (Ailanthus altissima Mill.), American pokeweed (Phytolacca americana L.) and common ragweed (Ambrosia artemisiifolia L.) are also frequent invasive species. 


\section{Acknowledgement}

I express my grateful thanks to Dr. Levente Ábrahám for reviewing, editing this paper and for his help in microscopic photography.

\section{References}

AchterberG, C. 2013: Hymenoptera in Fauna Europaea version 2.6.2. http://www.faunaeur.org. Last check: 20. 09. 2019

AchterberG, C. van \& B. van Aartsen 1986: The European Pamphiliidae (Hymenoptera: Symphyta), with special reference to The Netherlands. - Zoologische Verhandelingen Leiden 234: 1-98.

Blank, S. M., Hara, H., Mikulás, J., Csóka, G., Ciornei, C., Constantineanu, R., Constantineanu, I., Roller, L., Altenhofer, E., Huflejt \& T.; Vétek, G. 2010: Aproceros leucopoda (Hymenoptera, Argidae): An East Asian pest of elms (Ulmus spp.) invading Europe. - European Journal of Entomology, Ceské Budejovice 107: 357-367. https://doi.org/10.14411/eje.2010.045

Csóka, G., Mikulás, J., Blank, S. M., VÉTeK, G. 2010: A kanyargós szillevéldarázs (Aproceros leucopoda Takeuchi, 1939) megjelenése magyarországon. [First occurrence of the zigzag elm sawfly (Aproceros leucopoda Takeuchi, 1939) in Hungary.] - 1. In: KöMíves, T., Haltrich, A, Molnar, J. 56. Növényvédelmi Tudományos Napok. 2010. február 23-24. Budapest: i-xiv, A-D, 1-86.

Gyurkovics, H. \& Haris, A. 2012: Sawflies (Hymenoptera: Symphyta) from Szeged and its surroundings (SE Hungary). - Natura Somogyiensis 22: 163-182. https://doi.org/10.24394/NatSom.2012.22.163

Haris, A. 2001a: Revisional list of the Hungarian Nematinae with the description of three new species (Hymenoptera: Tenthredinidae). - Folia Entomologica Hungarica 62: 95-114.

HARIS, A. 2001b: Somogy megye levéldarázs-alkatúinak katalógusa (Hymenoptera, Symphyta). - Natura Somogyiensis 1: 261-268. https://doi.org/10.24394/NatSom.2001.1.261

HARIS, A. 2006: Study on the Palaearctic Pristiphora species (Hymenoptera: Tenthredinidae). - Natura Somogyiensis 9: 201-277. https://doi.org/10.24394/NatSom.2006.9.201

Haris, A. 2009: Sawflies of the Zselic Hills, SW Hungary (Hymenoptera: Symphyta). - Natura Somogyiensis 15: 127-158. https://doi.org/10.24394/NatSom.2009.15.127

Haris, A. 2010: Sawflies of the Vértes Mountains (Hymenoptera: Symphyta). - Natura Somogyiensis 17: 209-238. https://doi.org/10.24394/NatSom.2010.17.221

Haris, A. 2011: Sawflies of the Börzsöny Mountains (North Hungary) (Hymenoptera: Symphyta). - Natura Somogyiensis 19: 149-176. https://doi.org/10.24394/NatSom.2011.19.149

Haris, A. 2012: Sawflies of Belső-Somogy (Hymenoptera: Symphyta). - Natura Somogyiensis 22: 141-162. https://doi.org/10.24394/NatSom.2012.22.141

HARIS A. 2018a: Second contribution to the sawflies of Belső Somogy (Hymenoptera: Symphyta). - Natura Somogyiensis 31: 45-62. https://doi.org/10.24394/NatSom.2018.31.45

Haris A. 2018b: Sawflies from Külső-Somogy, South-West Hungary (Hymenoptera: Symphyta). - Natura Somogyiensis 32: 147-164. https://doi.org/10.24394/NatSom.2018.32.147

Lacourt, J. 2002: Révision des Caliroa Costa ouest-paléarctiques (Hymenoptera, Tenthredinidae). - Revue française d'Entomologie, (N. S.), Paris 24(3): 125-131.

Macek, J., Roller, L., Beneš, K. Holý, K. \& HolušA, J. 2020: Blanokřídlí České a Slovenské republiky II. Širopasí. - Academia Praha. 669 pp.

Mocsáry, S. 1900: Ordo Hymenoptera. p. 7-113. - In: Paszlavsky, J. (ed.): Fauna Regni Hungariae, Regia Societas Scientiarum Naturalium Hungarica, Budapest.

Prous, M.; Blank, S.; Goulet, H.; Heibo, E.; Liston, A.; Malm, T.; Nyman, T.; Schmidt, S.; Smith, D.; Vardal, H., Vittasaari, M., Vikberg, V. \& Taeger, A. 2014: The genera of Nematinae (Hymenoptera, Tenthredinidae). - Journal of Hymenoptera Research 40: 1-69. https://doi.org/10.3897/JHR.40.7442

Roller, L. 1993: New records of sawflies (Hymenoptera: Symphyta) from Slovakia. -Entomological Problems 24(2): 81-84.

Roller, L., 1994: Faunistics records. Symphyta. - Entomological Problems 25(2): 24.

Roller, L. 1996: New records of sawflies (Hymenoptera, Tenthredinidae) in Slovakia. - Biologia, Bratislava 51(1): 549-550.

Roller, L. 1998: Sawfly (Hymenoptera, Symphyta) community in the Devínska Kobyla National Nature Reserve. - Biologia, Bratislava 53(2): 213-221. 
Roller, L. 1999a: Spoločenstvá hrubopásych (Hymenoptera: Symphyta) vybraných zoogeografických regiónov Slovenska. - PhD thesis, Ústav zoológie, Slovenská akadémia vied, Bratislava, 180 pp.

Roller, L. 1999b: First records of Nematinae (Hymenoptera, Symphyta, Tenthredinidae) in Slovakia. Biologia, Bratislava 54(5): 599-600.

Roller, L. 1999c: Faunistic records from Slovakia. Hymenoptera: Symphyta: Tenthredinidae: Nematinae. Entomological Problems 30(1): 30.

Roller, L. 1999d: Faunistic records from Slovakia. Hymenoptera: Symphyta: Tenthredinidae: Nematinae. Entomological Problems 30(1): 52.

Roller, L., 1999e: Check list of the sawflies (Hymenoptera: Symphyta) of Slovakia. - Entomological Problems 30(2): 37-48.

Roller, L., 2000a Zubačkovité (Megalodontesidae) - hrubopáse xerotermov. - Hmyz 1(1): 17-18.

Roller, L., 2000b First records of Blasticotomidae, Tenthredinidae, Pamphiliidae (Hymenoptera) from Slovakia. - Biologia, Bratislava 55(5): 561-562.

Roller, L., 2000c Súčasný stav poznania fauny hrubopásych (Hymenoptera, Symphyta) na Slovensku. Správy Slovenskej zoologickej spoločnosti 18: 109-114.

Roller, L., 2001: Príspevok k poznaniu hrubopásych (Hymenoptera, Symphyta) a rohačkovitých (Diptera, Sciomyzidae) prírodnej pamiatky Mitická slatina. p. 32-36. - In: MáJSKY, J. (ed.), Zborník výsledkov inventarizačného výskumu prírodnej pamiatky Mitická slatina. Občianske združenie Pre Prírodu, Trenčín, $99 \mathrm{pp}$.

Roller, L. 2004. Hrubopáse blanokrídlovce (Hymenoptera, Symphyta) Tematínskych kopcov. - Entomofauna Carpathica 16: 56-64.

Roller, L., 2005: Blanokrídlovce (Hymenoptera): hrubopáse (Symphyta). 117-123 - In: Fauna Devínskej Kobyly. APOP, Bratislava, 181 pp.

Roller, L., 2006a: Seasonal flight activity of sawflies (Hymenoptera, Symphyta) in submontane region of the West Carpathians, Central Slovakia. - Biologia, Bratislava 61(2): 193-205. https://doi.org/10.2478/ s11756-006-0030-z

Roller, L., 2006b: Hrubopáse blanokrídlovce (Hymenoptera, Symphyta) Tematínskych vrchov - zhrnutie faunistického výskumu. p. 53-55. - In: K. RAJcová (ed.): Najvzácnejšie prírodné hodnoty Tematínskych vrchov. Zborník výsledkov inventarizačného výskumu územia európskeho významu Tematínske vrchy. KOZA, Trenčín a Pre Prírodu, Trenčín, 101 pp.

Roller, L. 2010. Hrubopáse blanokrídlovce (Hymenoptera: Symphyta) PR Š́r, pp. 215-235. In: MAJZLAN, O., VIDLIČKA, L'. (eds). - Príroda rezervácie Š́r. Ústav zoológie SAV, Bratislava, 410 pp.

Roller, L. \& Haris, A. 2008: Sawflies of the Carpathian Basin, History and Current Research. - Natura Somogyiensis 11. Kaposvár, 261. pp. https://doi.org/10.24394/NatSom.2008.11.2

Roller, L., Lukáš, J., 1999: New records of sawflies (Hymenoptera, Symphyta) in Slovakia. - Biologia, Bratislava 54(2): 225-228.

Roller L., Beneš K., Blank S. M., Holuša J., Jansen E., Jänicke M., Kaluza S., Kehl A., Kehr I., Kraus M., Liston A. D., Nyman T., Nie H., Savina H., Taeger A., Wei M., 2006: Contribution to the knowledge of sawfly fauna (Hymenoptera, Symphyta) of the Low Tatras National Park in Central Slovakia. - Naturae Tutela 10: 57-72.

Roller, L. \& MaceK, J. 2017: Provnález Hrubopásych Blanokrídlovcov (Hymenoptera, Symphyta) na Slovensku. - Entomofauna carpathica, 29(1): 53-63.

Roller, L. \& OLŠOvSKÝ, T. 2012: Prvonálezy hrubopásych blanokrídlovcov (Hymenoptera, Symphyta) v slatinných lesoch s tavol'níkom vŕbolistým (Spiraea salicifolia) v Borskej nížine. - Entomofauna carpathica 24(1): 15-20.

Taeger, A, Blank, S. M. \& Liston, A. 2006: European Sawflies (Hymenoptera: Symphyta). - A Species Checklist for the Countries. 399-504. - In Blank, S. M., Schmift, S. \& Taeger, A. (eds) Recent Sawfly Research: Synthesis and Prospects, Goecke \& Evers, Kelter. 701 pp.

Zhelochovtsev, A. N. 1988: Otryad Hymenoptera - Pereponchatokrylye, Podotryad Symphyta Sidyachebryukhie, 7-234. - In: Medvedev, K.H. (ed.) Opredelitel nasekomykh evropeiskoi chasti SSSR, Vol. 3 Hymenoptera, Part 6, Nauka, Leningrad.

Zombori, L. 1982: Tenthredinoidea - Levéldarázs-alkatúak II. - In: Fauna Hungariae, Akadémiai Kiadó, Budapest, 153, 11(3/A), $144 \mathrm{p}$.

Zombori, L. 1985: Adatok a Barcsi borókás növényevő darazsainak ismeretéhez (Hymenoptera, Symphyta). - Dunátúli Dolgozatok Természettudományi sorozat 2: 171-176.

Zombori, L. 2016: Levéldarázs-alkaúak IV. Tenthredinoidea IV. In Fauna Hungariae 9. Hymenoptera I. Fauna Hungariae 174. 3/c booklet. - Mondat Kft. and Hungarian Natural History Museum, Budapest. 160 pp. 
